\title{
Comportamento e intensidade dos ventos na zona costeira de Aquiraz - CE.
}

\author{
Behavior and intensity of the winds on the coast of Aquiraz-CE.
}

Daniel Rodrigues dos Santos danielrodri_8@hotmail.com Universidade de Fortaleza

Roquelina de Sousa de Saboya roquelinasaboya@gmail.com Universidade de Fortaleza

\begin{abstract}
Alexandre Farias Monte Monteiro

alexandremonteiro1986@ hotmail.com

Universidade de Fortaleza

Cibele Garcia Reis

cibele_garciareis@hotmail. com

Universidade de Fortaleza
\end{abstract}

Laís Sueanne Souza Teixeira lais.sueanne@gmail.com Universidade de Fortaleza

Lívia Castro Sales liviacsales@gmail.com Universidade de Fortaleza

Sofia Cavalcanti Lima de Holanda

sofiaclh@yahoo.com.br Universidade de Fortaleza

\section{Márcia Thelma Rios Donato Marino marino@unifor.br Universidade de Fortaleza}

Cledinaldo Castro Araújo cledinaldo1974@gmai.com Universidade de Fortaleza

\begin{abstract}
Resumo
A zona litorânea cearense é formada pela ação contínua dos ventos, caracterizando a presença de depósitos eólicos próximos à zona costeira, ambientes alvos da construção de núcleos residenciais, turístico-hoteleiros e comerciais. Essa ocupação desenfreada prejudica a dinâmica costeira, causando poluição dos aquíferos, alteração na morfologia dos campos de dunas, possíveis processos erosivos e assoreamento em estruturas naturais. Assim, é importante um estudo aprimorado dos processos físicos relacionados à sedimentação eólica, além de uma análise das frequências percentuais dos ventos, suas direções preferenciais e velocidades médias na região. A pesquisa de campo foi realizada no período chuvoso (Fevereiro/2016) utilizando-se um anemômetro digital e armadilhas de sedimentos. As interpretações indicaram que o fluxo de correntes eólicas de direção preferencial ENE corresponde à principal fonte dos depósitos eólicos que compõem a área de estudo. A análise do comportamento e velocidade dos ventos evidenciou um gradiente vertical ascendente de velocidade; certo padrão de comportamento das curvas traçadas em alturas diferenciadas; e maiores velocidades dos ventos na zona de estirâncio. A análise do comportamento dos ventos associada ao transporte eólico de sedimentos mostrou-se uma ferramenta essencial para compreender a dinâmica eólica e morfosedimentar, fornecendo dados para subsidiar a gestão costeira no que diz respeito ao seu uso e ocupação.
\end{abstract}

Palavras chaves: Zona litorânea. Corrente eólica. Dinâmicacosteira.

\begin{abstract}
The Ceará's coastal zone is formed by the continuous action of the winds, characterizing the presence of aeolian deposits near coastal zone, targets environments of building residential areas, tourist-hotel and commercial. This unbridled occupation affects coastal dynamics, causing pollution of aquifers, changes in the morphology of the dune fields, possible erosion and siltation in natural structures. Thus, a study of enhanced physical processes related to wind sedimentation is important, as well as an analysis of the percentage frequency of the winds, their preferred directions and average speeds in the region. The field research was carried out in the rainy season (February/2016) using a digital anemometer and sediment traps. The interpretations indicated that the flow of current wind preferred ENE direction corresponds to the major source of wind deposits that comprise the study area. The behavior analysis and wind speed showed an upward vertical gradient of velocity; certain pattern of behavior of the curves drawn in different heights; and higher wind speeds in foreshore zone. Behavior analysis of the winds associated with the wind sediment transport proved to be an essential tool to understand the wind and coastal dynamics, providing data to support coastal management with regard to its use and occupation.
\end{abstract}

Keywords: Coastal zone. Wind power. Coastal dynamics.

\section{Introdução}

A zona costeira consiste em uma região de relativa instabilidade, pois sofre alterações contínuas decorrentes de influências de diversos fatores como: sazonalidade, regime de marés, correntes marítimas, ventos, ação antrópica etc. Segundo Pasolini (2008) "a forma dos perfis de praia é o produto de um complexo sistema de forças que interagem entre si, formando um arcabouço estrutural dinâmico, que se modela à medida que as condições energéticas do ambiente se modificam".

O transporte de sedimentos em praias arenosas pode ser considerado em componentes transversal e longitudinal. A interação de processos transversais e longitudinais promove alterações contínuas nos perfis de praias de sedimentos não consolidados (PASOLINI, 2008). 
Daniel Rodrigues dos Santos, Roquelina de Sousa de Saboya, Alexandre Farias Monte Monteiro, Cibele Garcia Reis, Laís Sueanne Souza Teixeira, Lívia Castro Sales, Sofia Cavalcanti Lima de Holanda, Márcia Thelma Rios Donato Marino, Cledinaldo Castro Araújo

No litoral, principalmente em praias arenosas, é possível observar as mudanças dessa região através de estudos de transporte de sedimentos, fator de grande importância natural, pois sua dinamicidade reflete na contribuição de sedimentos para outros ecossistemas, no controle da zona de estirâncio, manutenção da faixa praial, bem como econômico-social, na degradação de patrimônios públicos e privados e outras, de acordo com Okamoto (2009). Ainda segundo esta autora, o estudo de transporte de sedimentos tem recebido especial atenção, pois é importante no planejamento de qualquer obra costeira que venha a interferir na dinâmica desse transporte para que se possam mitigar os danos ao meio ambiente e também às obras, que quando são executadas sem a devida precaução, podem sofrer com a deposição ou erosão de sedimentos e um consequente avanço do mar.

A interação entre os fluxos eólicos e fluxos de sedimentos depende em grande parte da velocidade do vento e das características granulométricas do material. Sua associação aos fluxos hidrodinâmicos é contribuição fundamental ao transporte de sedimentos ao longo de todo litoral do estado do Ceará (MORAIS et al., 2006). Segundo Oliveira e Meireles (2010) a ação eólica, a hidrodinâmica dos canais de maré e lagunas e a deriva litorânea foram os principais agentes modeladores do perfil litorâneo de Aquiraz.

Em regiões de grande interesse econômico, como é na grande parte do litoral brasileiro, é interessante o estudo de transporte de sedimentos para que possa haver um planejamento urbano de crescimento da cidade.

O local de estudo deste trabalho consiste em uma região de grande interesse turístico e imobiliário, a praia do litoral leste conhecida como Prainha, pertence ao município de Aquiraz, Ceará. Esta pequena cidade se localiza a uma média de 32,3 km do cento da capital do Ceará, registrando uma população que se aproximava dos 78.000 habitantes em 2015 (FORTALEZA, 2016). Segundo a Secretaria Estadual do Turismo de Fortaleza, Aquiraz é o segundo maior polo hoteleiro do estado do Ceará, fundamentado nos seus $28 \mathrm{~km}$ de praias (Porto das Dunas, Prainha, Presídio, Iguape, Barro-Preto e Batoque). Atualmente, vem sendo gradualmente ocupada por barracas, hotéis, e casas de veraneio (CÂMARA et al., 2010).

Prainha está situada na foz do rio Catu, a $5 \mathrm{~km}$ da sede do município de Aquiraz (Figura 1). Caracteriza-se por uma praia de morfodinâmica intermediária. Esse perfil morfodinâmico geralmente é caracterizado, segundo Calliari et al. (2003) por uma "progressiva redução da largura da calha longitudinal (longshoretrough), em decorrência da migração do banco submarino da zona de arrebentação em direção à praia o que por sua vez, é uma resposta às variações nas características hidrodinâmicas".

Figura 1: Localização da área de estudo.

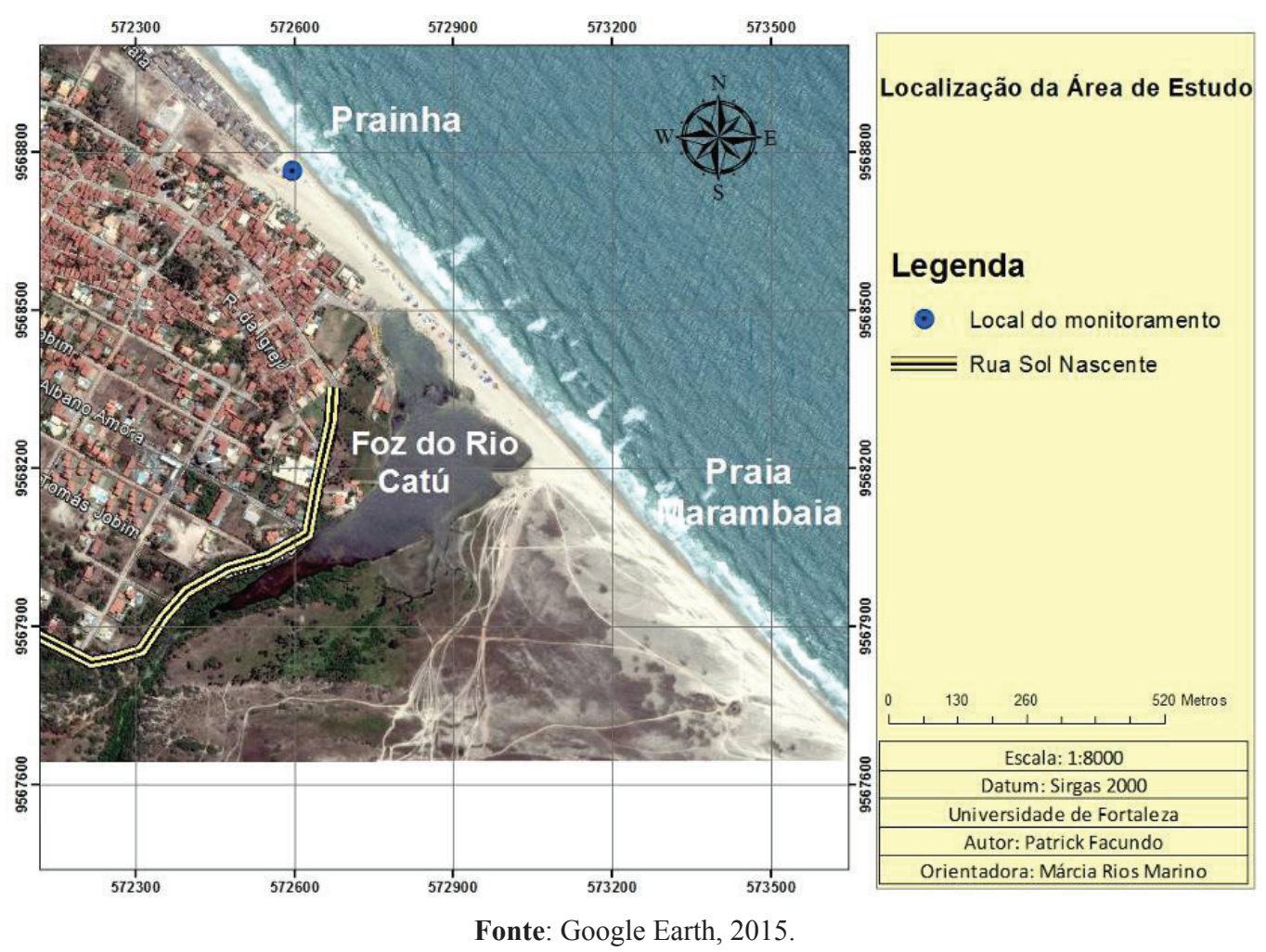


Diante dos fatos, a Prainha se encontra vulnerável diante da pressão do setor turístico e imobiliário, pois é reconhecida como uma bela praia beneficiada pela foz do rio, uma grande beleza natural como a cena do encontro do rio com o mar, e ainda sua proximidade com Fortaleza, estando sujeita assim, a processos erosivos que podem causar graves danos ao meio ambiente.

Este trabalho concentra-se na observação da dinâmica eólica de transporte de sedimentos na região da Prainha, pois se faz necessários estudos periódicos do tema para o acompanhamento do comportamento da dinâmica visando prever e dimensionar as alterações sazonais do regime de movimentação de sedimentos. Neste cenário, apresenta como objetivo determinar o comportamento das variações das correntes eólicas, durante os intervalos de tempo monitorados, em relação às alturas investigadas e ao volume de sedimentos transportados.

\section{Metodologia}

No dia 13 de fevereiro de 2016 foi realizada uma visita à Prainha, município de Aquiraz, com o objetivo de avaliar o transporte de sedimentos no período chuvoso de acordo com as correntes eólicas do local. Para o monitoramento foi utilizado um anemômetro digital portátil (MINIPA - MDA 11 / Precisão $\pm 3 \%$ ), e instalados dois grupos de armadilhas de sedimentos: um de acumulação (Figura 2), que acumula todo o material transportado através de uma seção vertical e outro grupo de discretização (Figura 3), que discretiza o transporte de sedimentos na vertical, discriminando-os em função da altura. As localizações geográficas de cada armadilha estão identificadas na Figura 4.

Figura 2 - Armadilhas de acumulação na zona de pós-praia, Fevereiro de 2016 (período chuvoso).

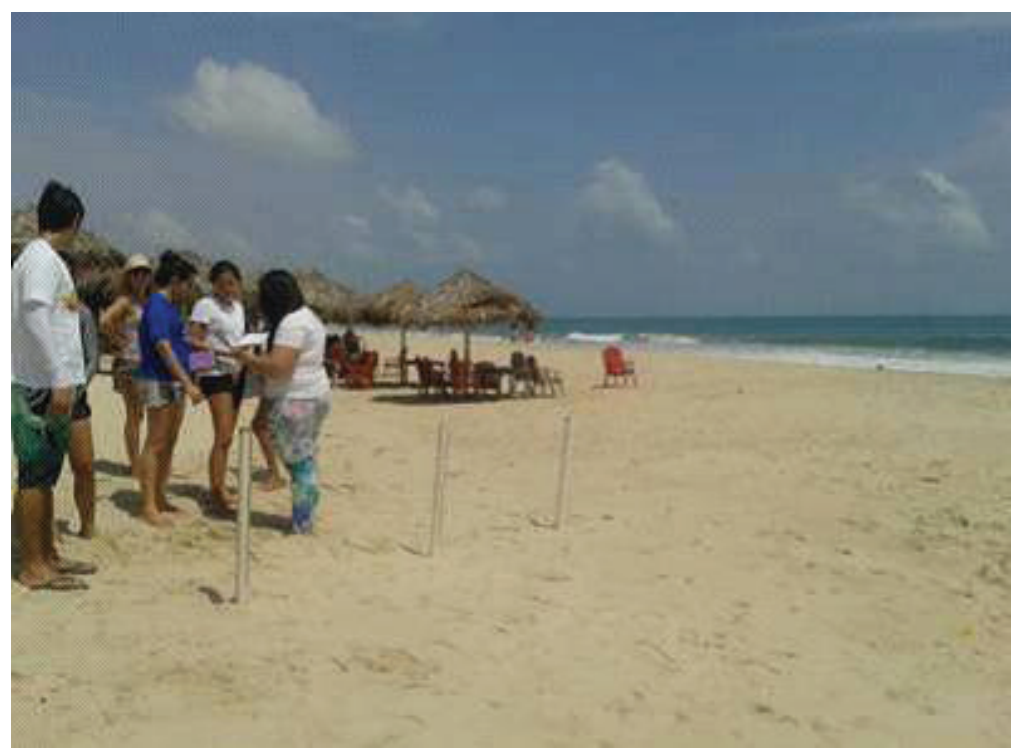

Fonte: Arquivo pessoal, 2016. 
Daniel Rodrigues dos Santos, Roquelina de Sousa de Saboya, Alexandre Farias Monte Monteiro, Cibele Garcia Reis, Laís Sueanne Souza Teixeira, Livia Castro Sales, Sofia Cavalcanti Lima de Holanda, Márcia Thelma Rios Donato Marino, Cledinaldo Castro Araújo

Figura 3 - Armadilhas de discretização na zona de estirâncio, Fevereiro de 2016 (período chuvoso).

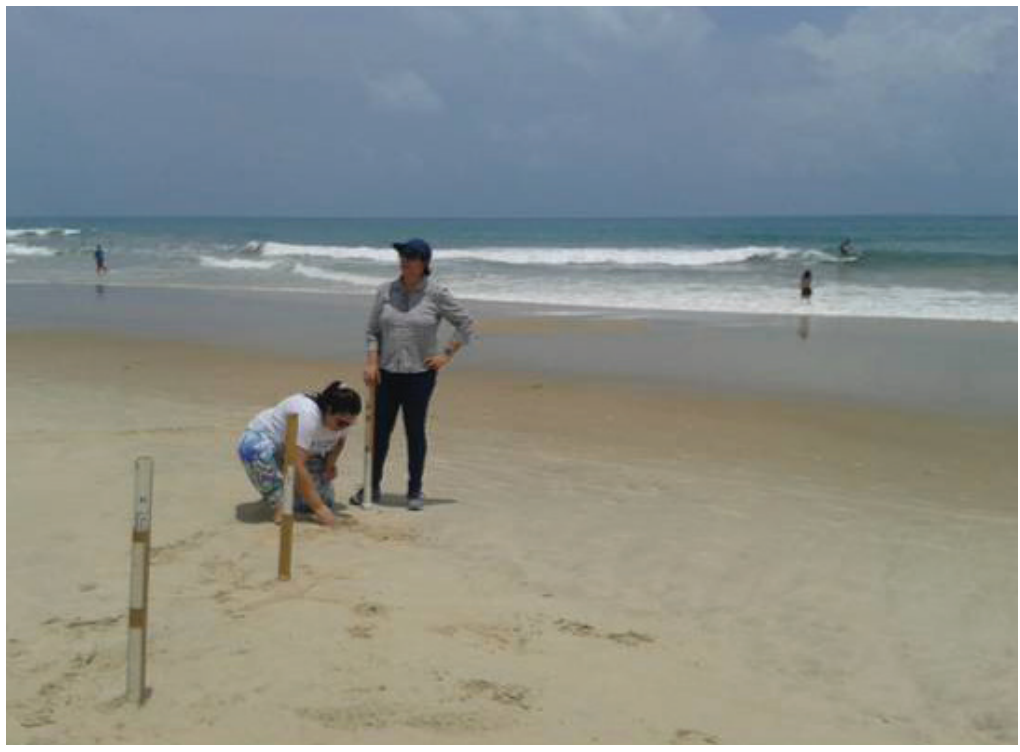

Fonte: Arquivo pessoal, 2016.

Figura 4 - Pontos geográficos das armadilhas de acumulação e discretização.

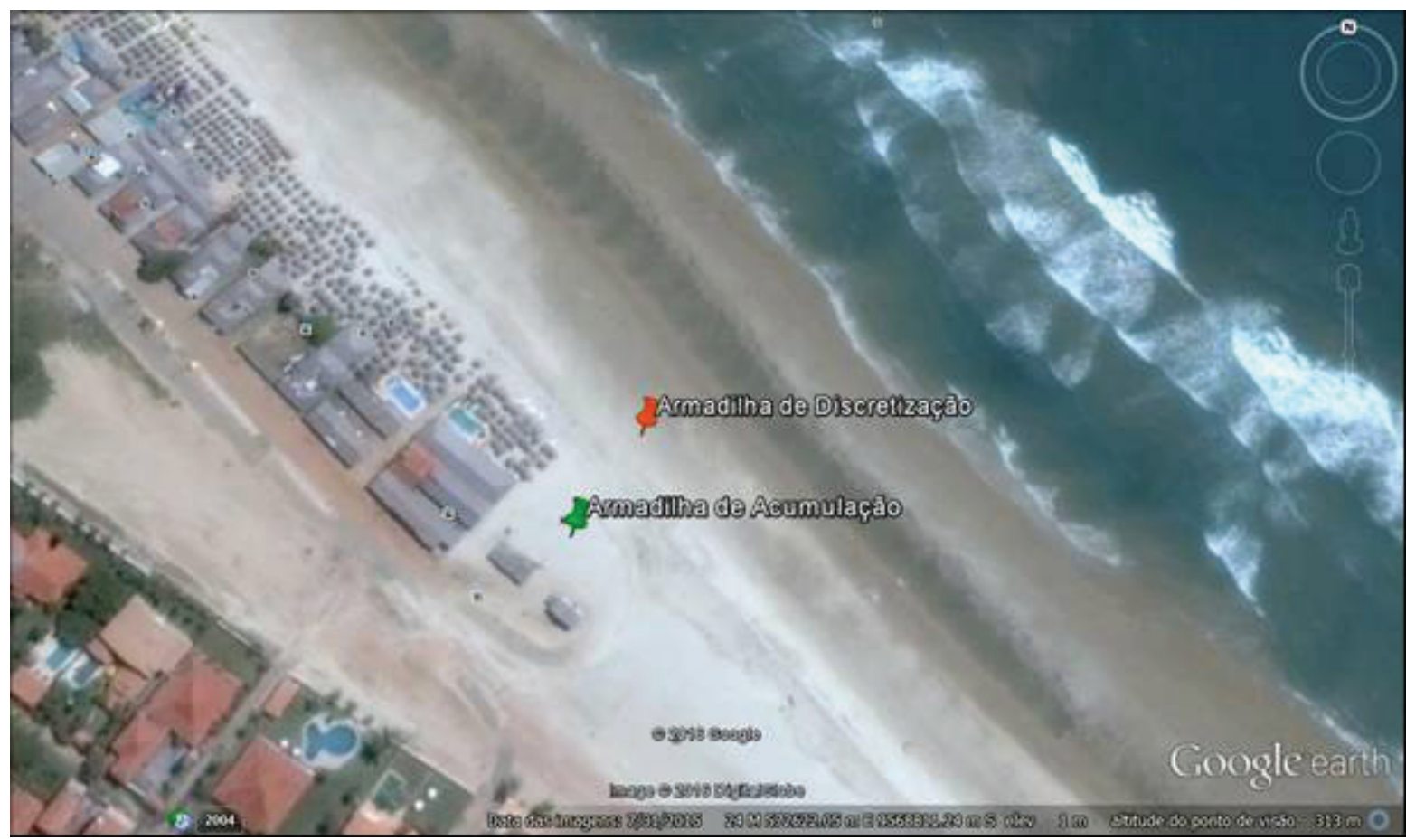

Fonte: Google Earth, 2016.

O monitoramento teve duração de três horas para cada grupo de armadilha, a de acumulação foi instalada na póspraia e a de discretização no estirâncio médio. $\mathrm{O}$ anemômetro foi utilizado seis vezes em cada armadilha nas alturas de 5, 25, 45, 100 e $200 \mathrm{~cm}$, nas quais ocorreram registros das velocidades momentâneas do vento (Figuras 5 e 6). Essas medições foram realizadas a cada 10 minutos, no período total de três horas. Previamente ao início dos trabalhos em campo, foi identificada a previsão das marés, como consta na Figura 7. 
Figura 5 - Medição da velocidade do vento nas armadilhas de acumulação na zona de pós-praia, Fevereiro de 2016 (período chuvoso).

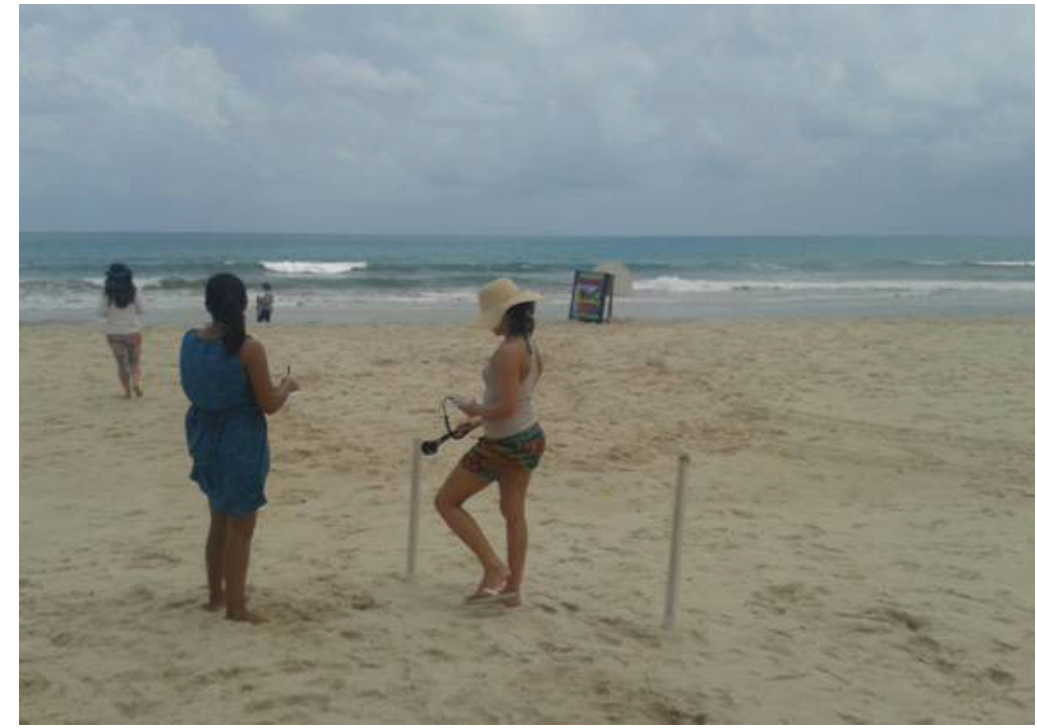

Fonte: Arquivo pessoal, 2016.

Figura 6 - Medição da velocidade do vento nas armadilhas de discretização na zona de estirâncio, Fevereiro de 2016 (período chuvoso).

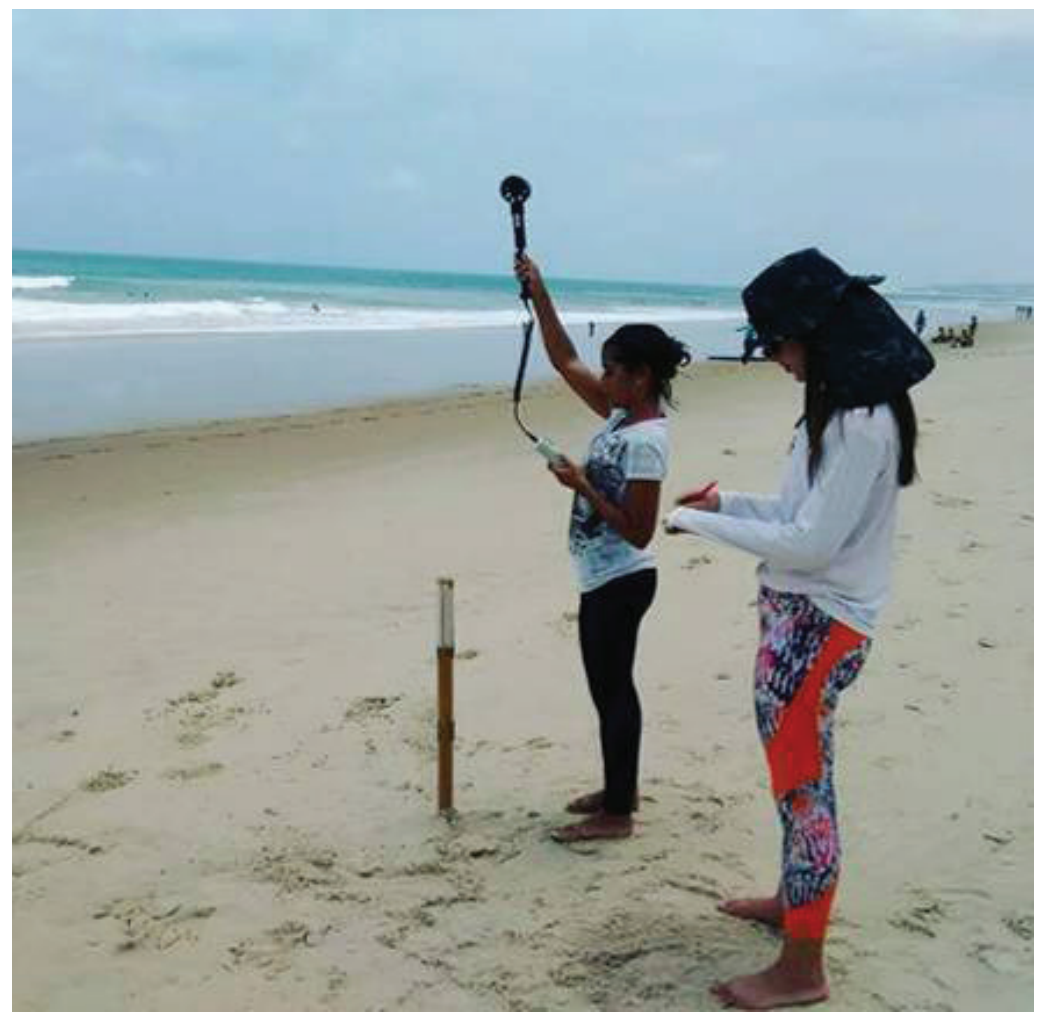

Fonte: Arquivo pessoal, 2016. 
Daniel Rodrigues dos Santos, Roquelina de Sousa de Saboya, Alexandre Farias Monte Monteiro, Cibele Garcia Reis, Laís Sueanne Souza Teixeira, Lívia Castro Sales, Sofia Cavalcanti Lima de Holanda, Márcia Thelma Rios Donato Marino, Cledinaldo Castro Araújo

Figura 7 - Previsão de marés obtida pelo CPTEC/INPE.

Fases da Lua

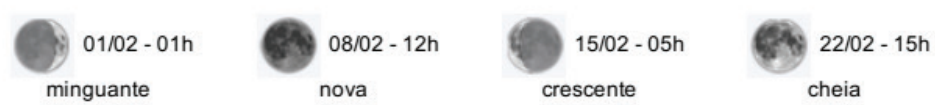

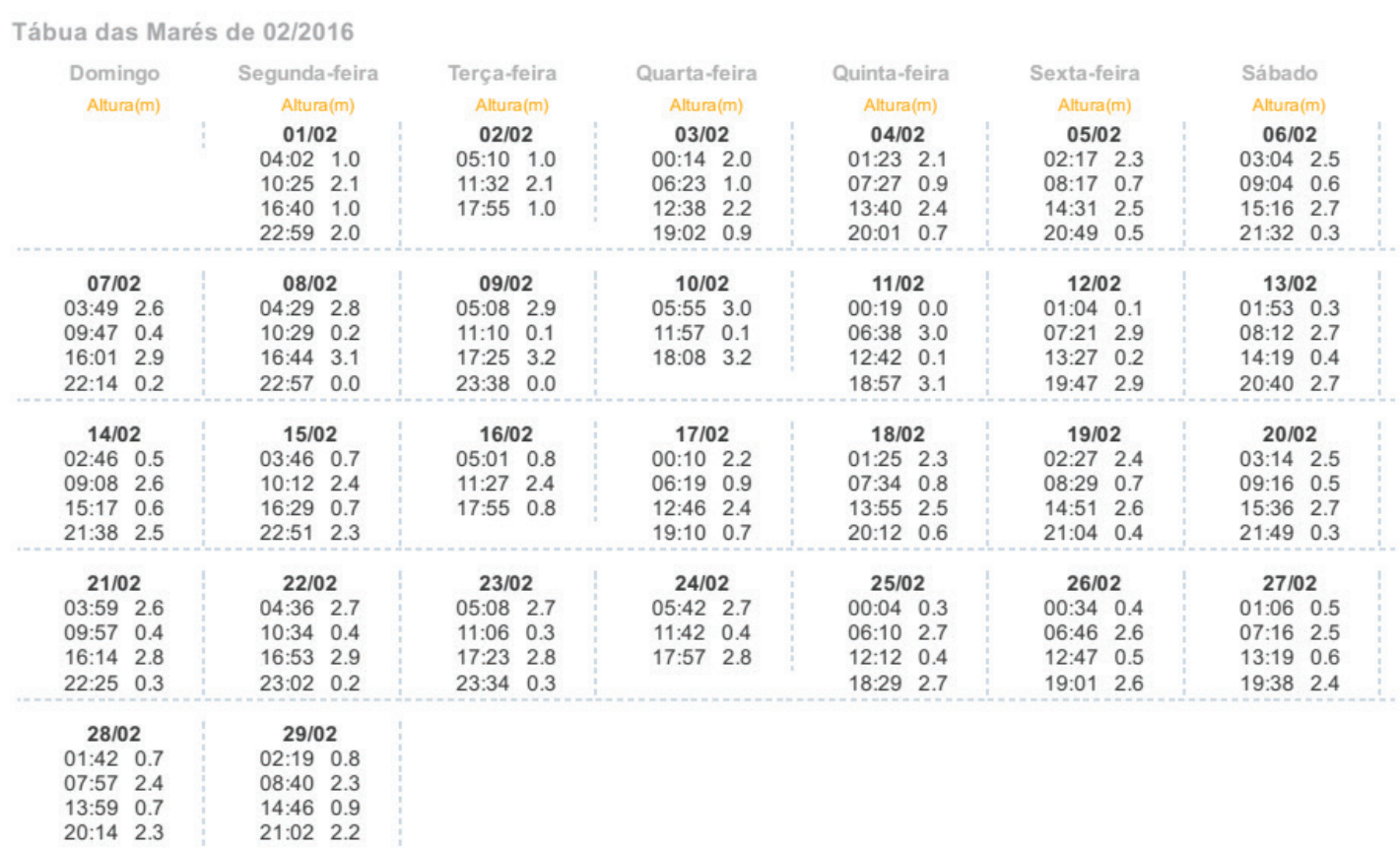

Fonte: CPTEC, 2016.

Foram utilizados dois tipos de armadilhas para análise do transporte de sedimentos. A armadilha de integração ou acumulação (posicionada na zona de pós-praia) acumula todo o material transportado na vertical e consiste em um tubo de PVC com $1 \mathrm{~m}$ de comprimento e $4 \mathrm{~cm}$ de diâmetro, com duas aberturas verticais em lados opostos, a frontal possui 70 $\mathrm{cm}$ de comprimento $\times 1 \mathrm{~cm}$ de largura e a posterior $70 \mathrm{~cm}$ de comprimento $\times 2,5 \mathrm{~cm}$ de largura.

A armadilha de discretização (posicionada no estirâncio médio) determina a distribuição vertical do transporte e das variações granulométricas dos sedimentos em função da velocidade do vento e da altura do transporte; e é formada por um tubo de PVC de $2 \mathrm{~cm}$ de diâmetro e $5 \mathrm{~cm}$ de comprimento, possuindo uma abertura frontal de $1 \mathrm{~cm}^{2}$ de área e uma posterior de $4 \mathrm{~cm}^{2}$ de área (FIGURAS 8a, 8b). As aberturas posteriores das armadilhas são recobertas por uma tela com malha de $63 \mathrm{~mm}$. 
Figura 8 - Desenho esquemático das armadilhas de sedimentos. (a) Integração (Acumulação) e (b) Discretização.

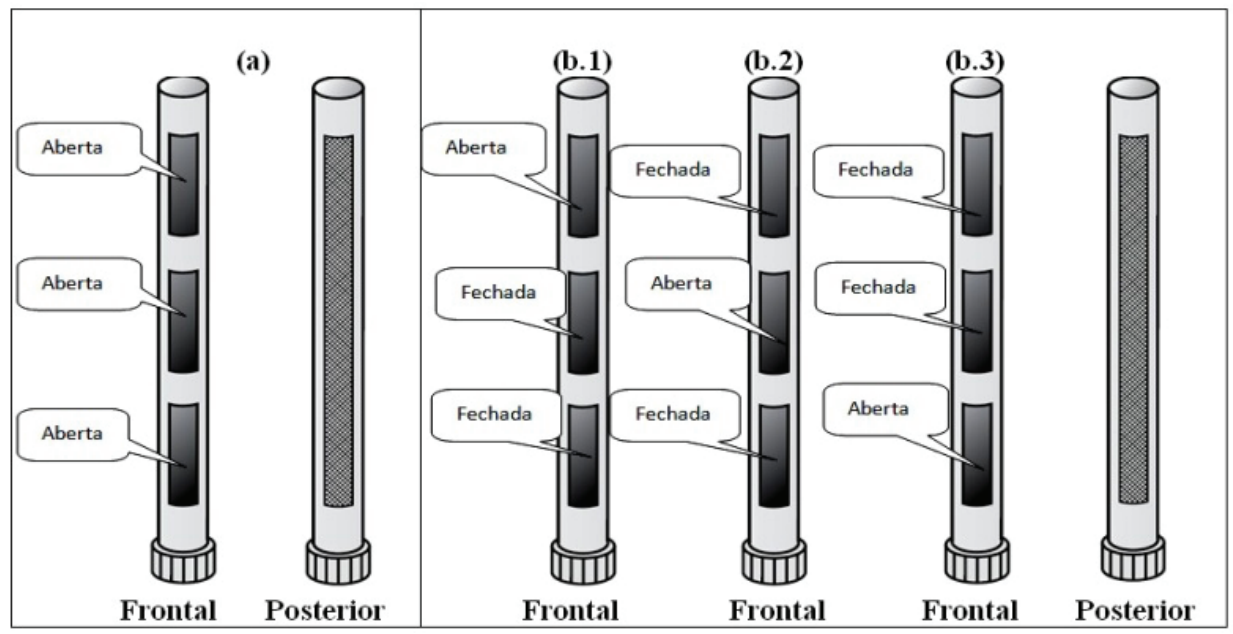

Fonte: Marino, 2014

Para análise dos dados foram feitos testes estáticos de comparação de média das velocidades dos ventos (T-student) e teste de variação das médias um a um (Shapiro-Wilk).

\section{Resultados e discussão}

As interpretações dos dados relacionados com as direções que os ventos atingem as células do litoral pesquisado e durante o período de amostragem, revelaram uma forte tendência de correntes oriundas do quadrante ENE, lestenordeste. ESE-E, correspondente aos ventos alísios, corroborando com os dados extraídos do NOAA Global Forecasting System e registrados no site Surfguru (Figuras 9 e 10).

Figura 9 - Velocidade vento médio superficial (m/s) e direção média do vento.

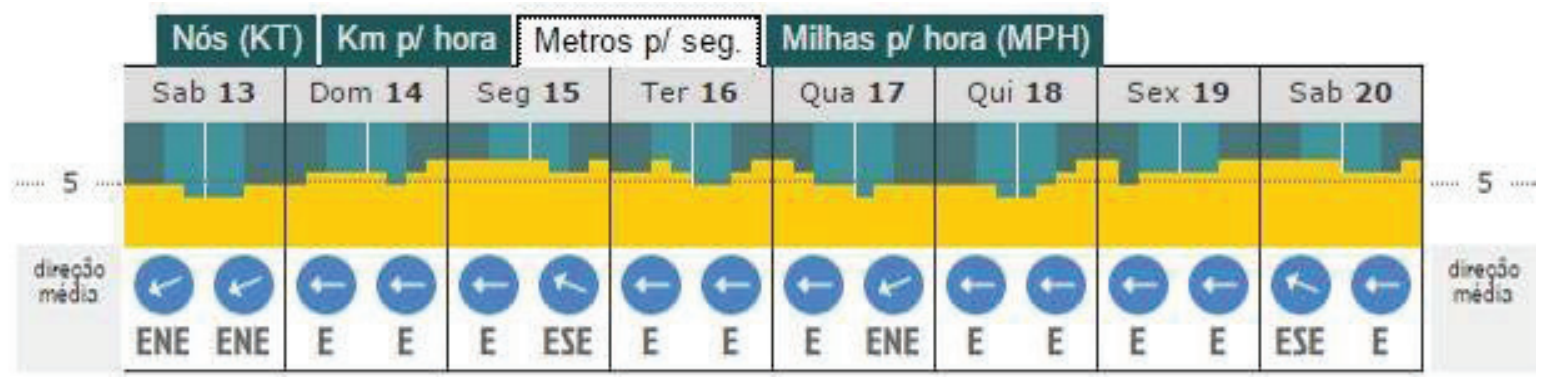

Fonte:Surfguru, 2016. 
Figura 10 - Direção média do vento e das correntes marítimas.
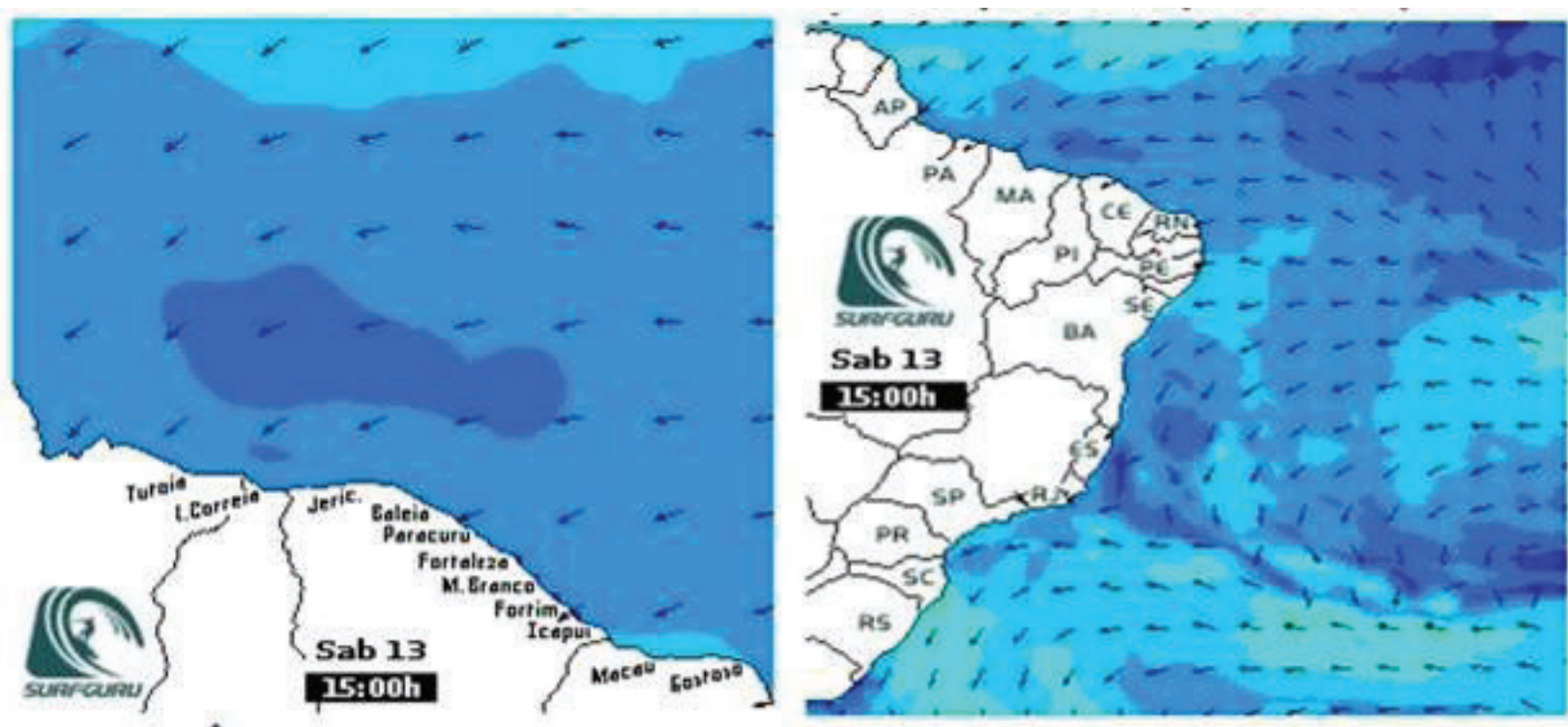

\begin{tabular}{|c|c|c|c|c|c|c|c|c|c|c|c|c|c|c|}
\hline $\begin{array}{l}9 \mathrm{~h} \\
\text { Sab }\end{array}$ & $\begin{array}{l}15 \mathrm{~h} \\
13\end{array}$ & $\begin{array}{l}9 \mathrm{~h} \\
\text { Dom }\end{array}$ & $\begin{array}{l}15 \mathrm{~h} \\
14\end{array}$ & $\begin{array}{l}\text { 9h } \\
\text { Seg }\end{array}$ & $\begin{array}{l}15 h \\
15\end{array}$ & $\begin{array}{l}\text { 9h } \\
\text { Ter }\end{array}$ & $\begin{array}{l}15 h \\
16\end{array}$ & $\begin{array}{l}9 \mathrm{~h} \\
\text { Qua }\end{array}$ & $\begin{array}{l}15 \mathrm{~h} \\
17\end{array}$ & $\begin{array}{l}\text { 9h } \\
\text { Qui }\end{array}$ & $\begin{array}{l}15 h \\
18\end{array}$ & $\begin{array}{l}\text { 9h } \\
\text { Sex }\end{array}$ & $\begin{array}{l}15 \mathrm{~h} \\
19\end{array}$ & $\begin{array}{ll}\text { 9h } & 15 h \\
\text { Sab } & 20\end{array}$ \\
\hline
\end{tabular}

Fonte:Surfguru, 2016.

Nos quadros 1 e 2,que se referem aos dados de frequência do vento obtidos nas armadilhas de discretização e acumulação, os pontos 1, 2, 3, 4 e 5 correspondem às alturas de 5, 25, 45, 100 e $200 \mathrm{~cm}$, respectivamente. Neles são observados que, nas armadilhas de discretização (Estirâncio Médio), as maiores velocidades médias das correntes de ar ocorreram a $45 \mathrm{~cm}$ e a $200 \mathrm{~cm}$ da superfície, registrando 3,09 m/sem ambas as alturas.Contudo,a máxima de velocidadefoi $4,24 \mathrm{~m} / \mathrm{s}$ a $25 \mathrm{~cm}$ da superfície e a mínima foi de $1,52 \mathrm{~m} / \mathrm{s}$ a $100 \mathrm{~cm}$ da superfície.

Quadro 1 - Dados de frequência do vento e transporte sedimentar eólico - Armadilha de discretização (Período chuvoso).

\begin{tabular}{|c|c|c|c|c|c|c|c|c|c|c|c|c|c|c|c|c|c|c|c|c|c|c|}
\hline \multicolumn{2}{|c|}{$\begin{array}{c}\text { Armadilha de } \\
\text { sedimento }\end{array}$} & \multicolumn{20}{|c|}{ Frequência Percentual do Vento } & \multirow{3}{*}{$\begin{array}{r}\begin{array}{c}\text { Sedime } \\
\text { aprision }\end{array} \\
\text { Volume }\end{array}$} \\
\hline $\mathbf{N}^{0}$ & \multirow[t]{2}{*}{ Posição } & \multicolumn{20}{|c|}{ Velocidade $(\mathrm{m} / \mathrm{s})$} & \\
\hline & & A & B & $\mathrm{C}$ & A & B & $\mathrm{C}$ & A & B & $\mathrm{C}$ & A & B & $\mathrm{C}$ & & 1 & B & $\mathrm{C}$ & A & B & $\mathrm{C}$ & \multirow{2}{*}{$\begin{array}{l}\text { Velocidade } \\
\text { Média }(\mathrm{m} / \mathrm{s})\end{array}$} & \\
\hline & Hora ${ }^{\circledR}$ & $11: 32$ & 11:37 & 11:43 & 12:02 & 12:07 & $12: 12$ & 12:31 & $12: 36$ & 12:4 & 13:0 & 13:0 & 13: & & $: 32$ & 13:37 & $13: 42$ & 14:04 & 14:09 & $14: 14$ & & (em 3 \\
\hline \multirow[t]{5}{*}{$\mathbf{I}$} & 1 & 1,97 & 2,94 & 2,89 & 3,02 & 3,33 & 3,11 & 2,85 & 3,61 & 2,99 & 1,9 & 2,7 & 3,2 & & 18 & 2,27 & 2,22 & 2,62 & 2,07 & 2,51 & 2,75 & \\
\hline & 2 & 2,61 & 2,48 & 2,63 & 3,37 & 3,04 & 2,96 & 2,42 & 3,35 & 4,24 & 1,8 & 2,8 & 2,6 & & 51 & 2,66 & 2,7 & 3,12 & 2,62 & 2,88 & 2,89 & \\
\hline & 3 & 2,8 & 3,02 & 2,83 & 3,8 & 3,67 & 3,69 & 2,81 & 3,03 & 3,88 & 1,9 & 2,9 & 3,1 & & 92 & 2,62 & 3,31 & 3,25 & 2,96 & 3,07 & 3,09 & \\
\hline & 4 & 2,35 & 2,59 & 2,91 & 3,2 & 2,8 & 4,03 & 2,51 & 2,75 & 3,72 & 1,5 & 2,9 & 3 , & & 09 & 3,35 & 2,83 & 2,62 & 3,82 & 2,7 & 2,94 & \\
\hline & 5 & 2,96 & 2,3 & 2,39 & 3,65 & 3,42 & 4,05 & 3,07 & 2,77 & 3,51 & 3,4 & 2,1 & 2,9 & & 09 & 2,38 & 2,79 & 3,16 & 3,31 & 4,16 & 3,09 & \\
\hline
\end{tabular}

Fonte: Elaborado pelos autores, 2016.

Baseado na estática de comparação das médias de velocidade dos ventos (T-student) as velocidades médias dos pontos 2 e 4 se mostraram estatisticamente iguais com o intervalo de confiança a $95 \%$. 
Quadro 2- Dados de frequência do vento e transporte sedimentar eólico - Armadilha de acumulação (Período chuvoso).

\begin{tabular}{|c|c|c|c|c|c|c|c|c|c|c|c|c|c|c|c|c|c|c|c|c|c|}
\hline \multicolumn{2}{|c|}{$\begin{array}{c}\text { Armadilha de } \\
\text { sedimento }\end{array}$} & \multicolumn{19}{|c|}{ Frequência Percentual do Vento } & \multirow{3}{*}{$\begin{array}{l}\begin{array}{c}\text { Sedime } \\
\text { aprision }\end{array} \\
\text { Volume }\end{array}$} \\
\hline$N$ & Posiçao & \multicolumn{19}{|c|}{ Velocidade $(\mathrm{m} / \mathrm{s})$} & \\
\hline & & $\mathbf{A}$ & B & $\mathrm{C}$ & A & B & $\mathrm{C}$ & A & B & $\mathrm{C}$ & $\mathbf{A}$ & B & $\mathrm{C}$ & $\mathbf{A}$ & B & $\mathrm{C}$ & A & B & $\mathrm{C}$ & \multirow{2}{*}{$\begin{array}{c}\text { Velocidade } \\
\text { Média }(\mathrm{m} / \mathrm{s})\end{array}$} & \\
\hline & Hora ${ }^{\circledR}$ & 10:18 & $10: 23$ & 10:28 & 10:47 & $10: 52$ & $10: 57$ & 11:16 & 11:21 & $11: 26$ & 11:46 & 11:51 & $11: 56$ & $12: 18$ & $12: 23$ & $12: 28$ & $12: 47$ & $12: 53$ & $12: 58$ & & \\
\hline \multirow[t]{5}{*}{ II } & 1 & 2,91 & 1,88 & 1,35 & 1,63 & 0,63 & 1,12 & 1,21 & 2,42 & 1,52 & 2,35 & 2,55 & 2,59 & 3,09 & 2,42 & 2,72 & 1,72 & 1,63 & 1,91 & 1,98 & \\
\hline & 2 & 2,89 & 1,91 & 1,83 & 0,9 & 1,45 & 1,34 & 2,2 & 2,68 & 1,41 & 2,12 & 1,62 & 2,72 & 2,18 & 2,94 & 2,83 & 1,95 & 2,45 & 2,52 & 2,11 & \\
\hline & 3 & 3,26 & 2,19 & 2,52 & 1,65 & 1,43 & 1,96 & 2,44 & 2,29 & 1,76 & 3,05 & 2,09 & 2,75 & 3,29 & 3,55 & 3,74 & 3,52 & 1,95 & 1,03 & 2,47 & \\
\hline & 4 & 3,24 & 1,74 & 1,61 & 2,66 & 0,56 & 0,32 & 2,14 & 2,81 & 2,31 & 3,63 & 2,38 & 2,94 & 3,44 & 3,63 & 3,74 & 0,7 & 2,08 & 2,43 & 2,35 & \\
\hline & 5 & 2,21 & 2,56 & 2,5 & 1,71 & 0,88 & 2,72 & 2,22 & 2,72 & 2,44 & 4,16 & 4,26 & 3,88 & 3,08 & 3,38 & 3,86 & 0,93 & 3,11 & 3,15 & 2,77 & \\
\hline
\end{tabular}

Fonte: Elaborado pelos autores, 2016.

Analisando as velocidades verificadas durante todo o monitoramento, as menores velocidades de correntes eólicas ocorreram na armadilha de acumulação, com menor valor registrado de $0,32 \mathrm{~m} / \mathrm{s}$. Contudo a maior velocidade registrada nesse período foi de $4,26 \mathrm{~m} / \mathrm{s}$ na zona de pós-praia (armadilha de acumulação), cuja medição ocorreu a uma altura de 200 cm da superfície, o que para Tomazelli (1990) corresponde à velocidade essencial para manter o fluxo dos sedimentos em movimento. Uma razão possível para justificar a maior velocidade neste ponto específico da armadilha de acumulação deve-se ao horário em que ocorreu sua medição, por volta de 12 a 13 horas da tarde, cujo período caracteriza-se por apresentar maior intensidade da radiação solar, a qual, através do aumento da temperatura, interfere na densidade do ar, tornando-o mais leve, portanto, facilitando o seu deslocamento (BARRETO et al., 2002).

Fazendo um comparativo entre as armadilhas de acumulação e discretização, utilizando-se das Figuras 11 e 12, verifica-se que as maiores médias de velocidade do ar se encontram no estirâncio médio (armadilha de discretização) tendo uma variação de $2,75 \mathrm{~m} / \mathrm{s}$ a $3,09 \mathrm{~m} / \mathrm{s}$. Com relação à velocidade média verificada nas armadilhas de acumulação, estão entre $1,98 \mathrm{~m} / \mathrm{s}$ e $2,77 \mathrm{~m} / \mathrm{s}$, sendo, portanto, as menores velocidades médias obtidas.

Figura 11 - Velocidade do vento na face de praia (Estirâncio médio) da Prainha. Período chuvoso (Fevereiro de 2016).

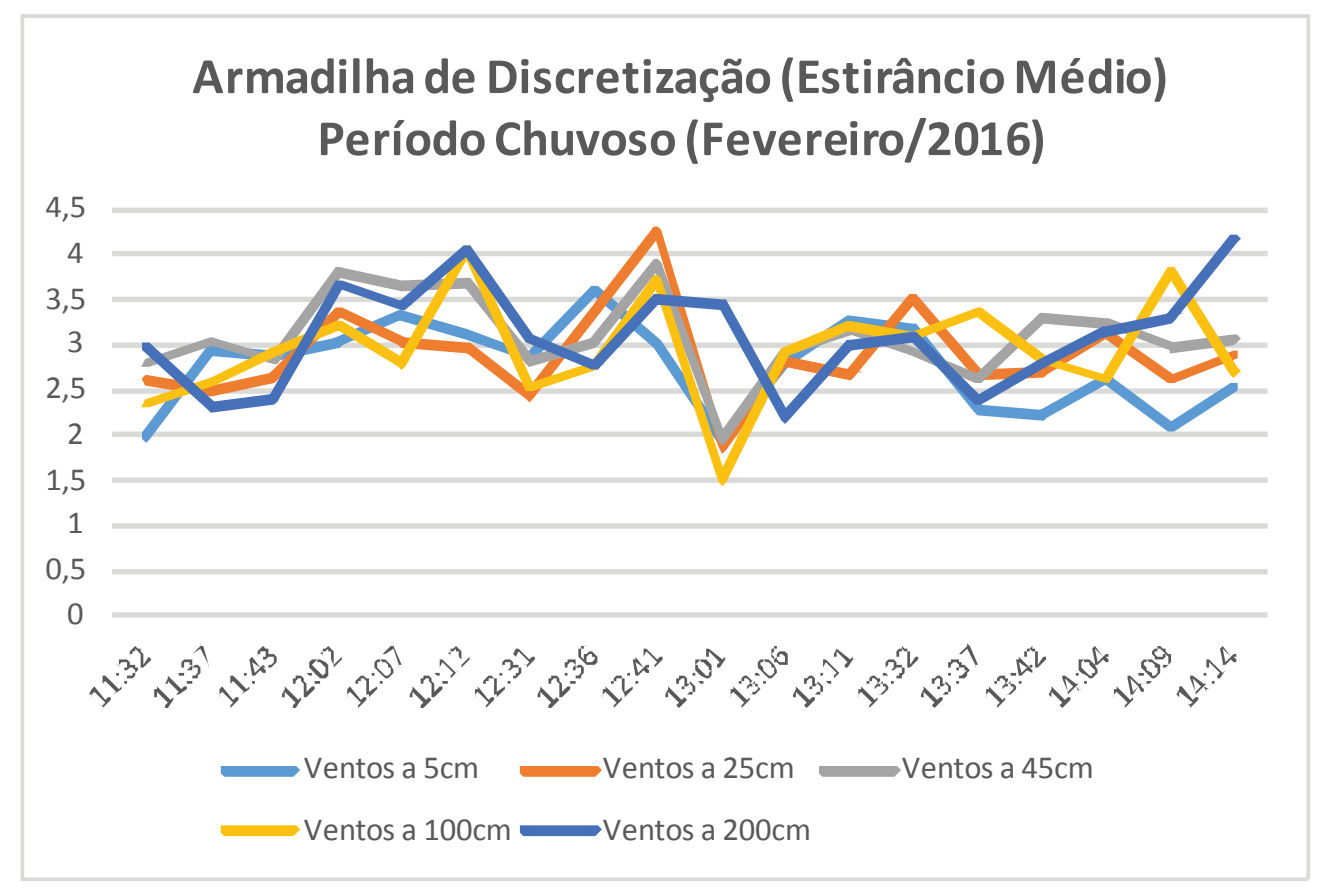

Fonte: Elaborado pelos autores, 2016. 
Figura 12 - Velocidade do vento na zona de pós-praia da Prainha. Período chuvoso (Fevereiro de 2016).

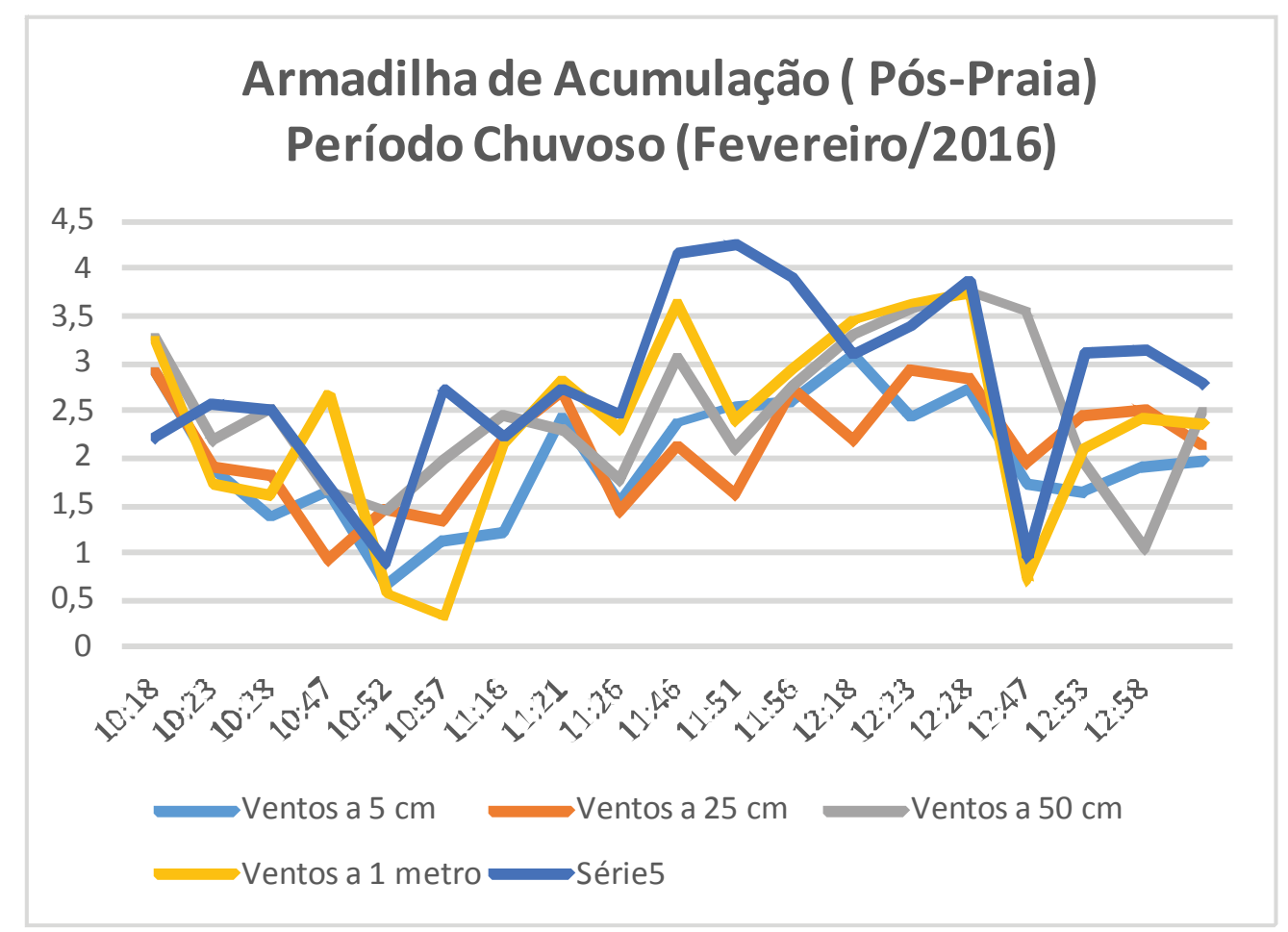

Fonte:Elaborado pelos autores, 2016.

A análise estatística gerada pelas médias das velocidades mostrou que não houve variâncias ponto a ponto, considerado um intervalo de confiança de $95 \%$.O padrão do aumento vertical do gradiente de velocidades das correntes de ar e que se mostra proporcional à altura, corrobora com as afirmações realizadas por Bagnold (1941) e Marino (2014), os quais relatam que a velocidade do vento diminui em função do atrito com a rugosidade do terreno e com a superfície dos grãos.

\section{Conclusão}

As observações das direções da dispersão dos grãos pelas correntes eólicas na zona de estirâncio (face de praia) permitiram verificar um maior volume do trânsito sedimentar litorâneo em direção à zona de pós-praia. Esse fato pode estar relacionado pela presença de dunas frontais posicionadas paralelamente à atual linha de costa.

O fluxo de correntes eólicas de direção preferencial ENE corresponde à principal fonte dos depósitos eólicos que compõem a área de estudo (faixa praial, campos de dunas e planície de deflação). Analisando os valores das velocidades das correntes de ar que circulam na região, é possível destacar alguns fatos: o desenvolvimento de um gradiente vertical ascendente de velocidade; certo padrão de comportamento das curvas traçadas em alturas diferenciadas; e maiores velocidades dos ventos na zona de estirâncio.

Por vezes, não existe conformidade entre as curvas obtidas nas diversas altitudes, fato que pode estar associado à coincidência do momento amostrado com episódios de oscilações bruscas das correntes eólicas, caracterizadas por picos de calmaria e agitação.

\section{Referências}

BAGNOLD, R. A. The physics of blown sand and desert dunes. London: Methuen, 1941. 265 p.

BARRETO, A. B., ARAGÃO, M. R. S.; BRAGA, C. C. Estudo do ciclo diário do vento à superfície no Nordeste do Brasil. In: CONGRESSO BRASILEIRO DE METEOROLOGIA, 12, 2002, Foz de Iguaçu. Anais... Foz do Iguaçu: 
CBMET.COM, 2002. p. 469-479. Disponível em:<http://www.cbmet.com/cbm-files/11-0185588409e90c7792bf8fcd83f 314a2.pdf>. Acesso em: 24 mar. 2016.

CALLIARI, L. J.; MUEHC, D.; HOEFEL, F. G.; TOLDO JR, E. Morfodinâmica praial: uma breve revisão (Beach morphodynamics: a brief review). Revista Brasileira de Oceanografia, v. 51, p. 63-78, 2003. Disponível em: <http:// www.scielo.br/pdf/rboce/v51nunico/07.pdf>. Acesso em: 24 mar. 2016.

CÂMARA, C. de F.; LOURENÇO, R. M.; FARIAS, J. F.; SILVA, E. V. Estudo geoambiental do litoral da prainha e do Porto das Dunas/ Aquiraz - CE: propostas para o planejamento ambiental. In: SEMINÁRIO LATINO AMERICANO DE GEOGRAFIA FÍSICA, 6, 2010, Coimbra; SEMINÁRIO IBERO AMERICANO DE GEOGRAFIA FÍSICA, 2, 2010, Coimbra, Actas... Coimbra: SLAGF, 2010. Disponível em: <http://www.uc.pt/fluc/cegot/VISLAGF/actas/tema3/ camila_freitas>. Acesso em: 10 de abr. 2016.

FORTALEZA. Secretaria Municipal do Turismo de Fortaleza. Distâncias. Disponível em: <http://www.fortaleza. ce.gov.br/turismo/distancias>. Acesso em: 23 mar. 2016.

GOOGLE EARTH. Software google earth.: sistema operacional Microsoft Windows (6.1.7601.1). 2010. Disponível em: <http://www.google.com.br/earth/download/ge/agree.html>. Acesso em: 9 mar. 2016.

INSTITUTO NACIONAL DE PESQUISAS ESPACIAIS. Previsão oceânica. Disponível em: <http://ondas.cptec.inpe. br/>. Acesso em: 13 fev. 2016.

MARINO, M. T. R. D. Análise integrada dos aspectos geoambientais e da dinâmica costeira de médio e curto periodo da planície litorânea entre as praias do Futuro e Porto das Dunas, Ceará, Brasil. 2014. 273 f. Tese (Doutorado) Centro de Ciências, Universidade Federal do Ceará. Fortaleza, 2014.

MORAIS, J. O. de. et al. Caracterização fisiográfica e geoambiental da zona costeira do estado do Ceará. In: MUEHE, Dieter (Org.). Erosão e Progradação do Litoral Brasileiro. Brasília, DF: Ministério do Meio Ambiente (MMA), 2006. p. 131-154. Disponível em: <http://www.mma.gov.br/estruturas/sqa_sigercom/_arquivos/ce_erosao.pdf $>$. Acesso em: 13 abr. 2016.

OKAMOTO, N. Transporte de sedimentos e estado morfodinâmico da praia da curva de Jurema, Vitória - ES. 2009. 97f. Trabalho de Conclusão de Curso (Graduação em Oceonografia) - Departamento de Oceanografia e Ecologia. Universidade Federal do Espírito Santo. Vitória, 2009. Disponível em: <http://www.oceanografia.ufes.br/sites/ oceanografia.ufes.br/files/field/anexo/NAYLA\%20OKAMOTO.pdf>. Acesso em: 27 abr. 2016.

OLIVEIRA, G. G.; MEIRELES, A. J. de A. Dinâmica geoambienal a partir da "litoralização" de Aquiraz, Ceará, Brasil. REDE - Revista Eletrônica do Prodema, Fortaleza, v. 5, n. 2, p. 50-68, jun. 2010. Semestral. Disponível em: <http:// www.revistarede.ufc.br/revista/index.php/rede/article/view/78/33>. Acesso em: 27 abr. 2016.

PASOLINI, A. Utilização do método de perfil de equilibrio para determinar a estabilidade e a evolução de perfis de praias arenosas. 2008. 137f. Dissertação (Mestrado) - Centro Tecnológico, Universidade Federal do Espírito Santo,Vitória, 2008. Disponível em:<http://portais4.ufes.br/posgrad/teses/tese_2712_UTILIZA\%C7\%C3O\%20 DO\%20M\%C9TODO\%20DE\%20PERFIL\%20DE\%20EQUIL\%CDBRIO\%20PARA \%20DETERMINAR\%20A\%20 ESTABILIDADE\%20E\%20A\%20EVOLU\%C7\%C3O\%20DE\%20PRAIA.pdf >. Acesso em: 27 abr. 2016.

SURFGURU. Central de previsão. Disponível em:<http://www.surfguru.com.br/previsao>. Acesso em: 13 fev. 2016.

TOMAZELLI, L. J. Contribuição ao estudo dos sistemas deposicionais holocênicos do nordeste da província costeira do Rio Grande do Sul - com ênfase no sistema eólico. 1990. 270 f. Tese (Doutorado) - Universidade Federal do Rio Grande do Sul, Porto Alegre, 1990. 
Daniel Rodrigues dos Santos, Roquelina de Sousa de Saboya, Alexandre Farias Monte Monteiro, Cibele Garcia Reis, Laís Sueanne Souza Teixeira, Lívia Castro Sales, Sofia Cavalcanti Lima de Holanda, Márcia Thelma Rios Donato Marino, Cledinaldo Castro Araújo

Sobre os autores

\section{Daniel Rodrigues dos Santos}

Cientista Ambiental, Universidade Federal do Ceará - UFC. Mestrado em Recursos Naturais, área de Biotecnologia, Universidade Estadual do Ceará- UECE. Especializando (a) em Gestão Ambiental, Universidade de Fortaleza - UNIFOR.

\section{Roquelina de Sousa de Saboya}

Bióloga, Universidade Estadual do Ceará- UECE.Especializando (a) em Gestão Ambiental, Universidade de Fortaleza UNIFOR.

\section{Alexandre Farias Monte Monteiro}

Biólogo, Universidade Federal do Ceará- UFC.Especializando (a) em Gestão Ambiental, Universidade de Fortaleza UNIFOR.

\section{Cibele Garcia Reis}

Engenheira Ambiental, Faculdade de Ciências e Tecnologias da Bahia - Área 1.Analista de recursos hídricos da Companhia de Gestão de Recursos Hídricos do Ceará- COGERH.Especializando (a) em Gestão Ambiental, Universidade de Fortaleza - UNIFOR.

\section{Laís Sueanne Souza Teixeira}

Bióloga, Universidade Estadual do Ceará- UECE.Especializando (a) em Gestão Ambiental, Universidade de Fortaleza UNIFOR.

\section{Lívia Castro Sales}

Bióloga, Universidade Estadual do Ceará- UECE.Especializando (a) em Gestão Ambiental, Universidade de Fortaleza UNIFOR.

\section{Sofia Cavalcanti Lima de Holanda}

Bióloga, Universidade Federal do Ceará- UFC.Especializando (a) em Gestão Ambiental, Universidade de Fortaleza UNIFOR.

\section{Márcia Thelma Rios Donato Marino}

Geóloga, Universidade de Fortaleza - UNIFOR.Especialização em Aperfeiçoamento em Mapeamento Geológico, Universidade Federal de Minas Gerais - UFMG. Mestrado em Geologia, Universidade Federal do Ceará - UFC. Doutorado em Geologia,Universidade Federal do Ceará - UFC. Coordenadora do curso de Especialização em Gestão Ambiental e do MBA em Perícia e Auditória Ambiental da Universidade de Fortaleza - UNIFOR. Professora Assistente da Universidade de Fortaleza - UNIFOR do curso de Graduação em Engenharia Ambiental e Sanitária.

\section{Cledinaldo Castro Araújo}

Graduado em Estatística pela Universidade Federal do Ceará - UFC (2002) e mestrado em Logística e Pesquisa Operacional pela Universidade Federal do Ceará - UFC (2012). Atualmente é professor da Universidade de Fortaleza UNIFOR. 